\title{
Nuclear overexpression of lymphoid- enhancer-binding factor 1 identifies chronic lymphocytic leukemia/small lymphocytic lymphoma in small B-cell lymphomas
}

\author{
Bevan Tandon ${ }^{1}$, LoAnn Peterson ${ }^{1}$, Juehua Gao ${ }^{1}$, Beverly Nelson ${ }^{1}$, Shuo $\mathrm{Ma}^{2}$, \\ Steven Rosen ${ }^{2}$ and Yi-Hua Chen ${ }^{1}$ \\ ${ }^{1}$ Division of Hematopathology, Department of Pathology, Northwestern University Feinberg School of \\ Medicine, Chicago, IL, USA and ${ }^{2}$ Division of Hematology/Oncology, Department of Internal Medicine, \\ Northwestern University Feinberg School of Medicine, Chicago, IL, USA
}

\begin{abstract}
Lymphoid-enhancer-binding factor 1 (LEF1), coupling with $\beta$-catenin, functions as a key nuclear mediator of WNT/ $\beta$-catenin signaling, which regulates cell proliferation and survival. LEF1 has an important role in lymphopoiesis, and is normally expressed in $T$ and pro-B cells but not mature B cells. However, gene expression profiling demonstrates overexpression of LEF1 in chronic lymphocytic leukemia, and knockdown of LEF1 decreases the survival of the leukemic cells. So far, the data on LEF1 expression in B-cell lymphomas are limited. This study represents the first attempt to assess LEF1 by immunohistochemistry in a large series (290 cases) of B-cell lymphomas. Strong nuclear staining of LEF1 was observed in virtually all neoplastic cells in 92 of $92(100 \%)$ chronic lymphocytic leukemia/small lymphocytic lymphomas including two CD5- cases, with strongest staining in cells with Richter's transformation. LEF1 also highlighted the morphologically inconspicuous small lymphocytic lymphoma component in three composite lymphomas. All 53 mantle cell lymphomas, 31 low-grade follicular lymphomas and 31 marginal zone lymphomas, including 3 CD5 + cases, were negative. In 12 grade 3 follicular lymphomas, LEF1 was positive in a small subset (5-15\%) of cells. Diffuse large B-cell lymphoma, however, demonstrated significant variability in LEF1 expression with overall positivity in 27 of $71(38 \%)$ cases. Our results demonstrate that nuclear overexpression of LEF1 is highly associated with chronic lymphocytic leukemia/small lymphocytic lymphoma, and may serve as a convenient marker for differential diagnosis of small B-cell lymphomas. The expression of $\beta$-catenin, the coactivator of LEF1 in WNT signaling, was examined in $\mathbf{5 0}$ chronic lymphocytic leukemia/small lymphocytic lymphomas, of which $\mathbf{4 4}$ $(88 \%)$ showed negative nuclear staining. The findings of universal nuclear overexpression of LEF1 but lack of nuclear $\beta$-catenin in the majority of chronic lymphocytic leukemia/small lymphocytic lymphoma suggest that the pro-survival function of LEF1 in this disease may be independent of WNT/ $\beta$-catenin signaling.

Modern Pathology (2011) 24, 1433-1443; doi:10.1038/modpathol.2011.103; published online 17 June 2011
\end{abstract}

Keywords: $\beta$-catenin; chronic lymphocytic leukemia/small lymphocytic lymphoma; diffuse large B-cell lymphoma; follicular lymphoma; lymphoid-enhancer-binding factor 1; mantle cell lymphoma; marginal zone lymphoma

Chronic lymphocytic leukemia is the most common leukemia in adults in the Western countries; small

Correspondence: Dr Y-H Chen, MD, Division of Hematopathology, Department of Pathology, Northwestern University Feinberg School of Medicine, 251 East Huron, Feinberg 7-209A, Chicago, IL 60611, USA.

E-mail: y-chen5@northwestern.edu

Received 7 April 2011; revised 26 April 2011; accepted 3 May 2011; published online 17 June 2011 lymphocytic lymphoma is the tissue counterpart of the disease. ${ }^{1}$ Chronic lymphocytic leukemia/small lymphocytic lymphoma is characterized by a progressive, clonal expansion of CD5 + B cells in the peripheral blood, bone marrow or lymph node. The expansion of the B cells was initially thought to be secondary to the prolonged survival due to the defects in apoptosis. Current data suggest that increased proliferation also contributes to the development of chronic lymphocytic leukemia/small lymphocytic lymphoma. ${ }^{2}$ 
Several recent studies have demonstrated dysregulation of $\mathrm{WNT} / \beta$-catenin signaling in chronic lymphocytic leukemia. ${ }^{3-7}$ This pathway regulates cell fate determination during development as well as cell proliferation and survival. The ultimate mediator of this pathway is a nuclear complex of LEF/T-cell factor (TCF) and $\beta$-catenin that work together to regulate the transcription of a variety of WNT target genes. ${ }^{8-10}$ Lymphoid-enhancer-binding factor 1 (LEF1) belongs to the LEF/TCF family that is comprised of four transcriptional factors, TCF1, LEF1, TCF3 and TCF $4 .{ }^{9}$ LEF1 is normally expressed in $\mathrm{T}$ and pro-B cells, and is involved in early lymphocyte development. ${ }^{11-15}$ LEF1-deficient mice exhibit defects in pro-B cell survival and proliferation; the increased proliferation of pro-B cells induced by WNT signaling is dependent on LEF1. ${ }^{11}$ The expression of LEF1 is turned off in mature B cells; however, gene expression profiling has revealed overexpression of LEF1 in chronic lymphocytic leukemia/small lymphocytic lymphoma in comparison with normal B cells. ${ }^{16,17}$ A recent study demonstrated that LEF1 is not only overexpressed in chronic lymphocytic leukemia/small lymphocytic lymphoma, but also in its preleukemic state of monotypic B lymphocytosis. ${ }^{7}$ These findings suggest that LEF1 may have a role in the pathogenesis of chronic lymphocytic leukemia.

So far, the data on LEF1 expression in B-cell lymphoma are limited, and the evaluation has been primarily through molecular or western blot analysis. ${ }^{3-7}$ Our study represents the first attempt to assess LEF1 expression by immunohistochemistry that allows us to evaluate a large series of chronic lymphocytic leukemia/small lymphocytic lymphoma as well as other types of B-cell lymphomas. Given the central role of LEF/TCF in $\mathrm{WNT} / \beta$-catenin signaling, the expression of $\beta$-catenin was also examined in order to investigate whether the pro-survival effect of LEF1 in chronic lymphocytic leukemia/small lymphocytic lymphoma is through mediating nuclear response to WNT signaling.

\section{Materials and methods}

\section{Case Selection}

This study was approved by the Institutional Review Board of the Northwestern Memorial Hospital, Chicago, IL, USA. Cases diagnosed as chronic lymphocytic leukemia/small lymphocytic lymphoma, mantle cell lymphoma, follicular lymphoma, marginal zone lymphoma and diffuse large B-cell lymphoma between January 2002 and November 2010 were searched and retrieved from the database in the Department of Pathology at the Northwestern Memorial Hospital. The H\&E- and immunohistochemical-stained slides as well as the results of ancillary studies were re-reviewed to confirm the diagnosis based on the current WHO classification. ${ }^{1}$ A total of 290 cases of B-cell lymphomas were randomly selected for the study, including 92 chronic lymphocytic leukemia/small lymphocytic lymphomas, 53 mantle cell lymphomas, 43 follicular lymphomas, 31 marginal zone lymphomas and 71 diffuse large B-cell lymphomas. Among these cases, three were composite lymphomas including one case of composite small lymphocytic lymphoma and mantle cell lymphoma and two cases of composite small lymphocytic lymphoma and follicular lymphoma.

\section{Immunohistochemistry and Flow Cytometric Immunophenotyping}

Immunohistochemical stains were performed on whole paraffin-embedded tissue sections. Antigen retrieval and immunohistochemical stain were performed on automated Leica Bond Max (Leica Microsystems, IL, USA). The primary antibodies used were as follows: monoclonal anti-LEF1 at a dilution of 1:100 for formalin-fixed tissue or 1:25 for B5-fixed bone marrow core (clone: EPR2029Y; Epitomics, Burlingame, CA, USA), monoclonal anti- $\beta$-catenin at a dilution of 1:400 for formalinfixed tissue or 1:100 for B5-fixed bone marrow core (clone: B-catenin-1, Dako), monoclonal anti-CD20, ready to use (clone: MJ1, Leica) and monoclonal anti-CD3, ready to use (clone: LN10, Leica). Immunohistochemical stain for cyclin D1 was previously performed in all mantle cell lymphomas included in this study. ${ }^{18}$ Reactive tonsillar tissues and reactive lymph nodes were used as controls.

Flow cytometric immunophenotyping was performed at the time of diagnosis as described previously. ${ }^{19}$

\section{Results}

\section{LEF1 Expression in Normal Lymphoid Tissue}

In normal lymphoid tissue, nuclear staining of LEF1 was seen predominantly in the cells in the paracortical regions. Double staining of LEF1 with CD3 demonstrated co-staining of nuclear LEF1 and cytoplasmic CD3 in the same cells, indicating that the T cells were positive for LEF1 but not the B cells (Figure 1). Variable numbers of LEF1 + T cells were also seen in the germinal centers. Double staining of LEF1 with CD20 showed rare B cells in the germinal centers were weakly positive for LEF1. In addition, some of the endothelial cells were also weakly positive for nuclear LEF1.

\section{LEF1 Expression in B-Cell Lymphomas}

Immunohistochemical staining for LEF1 was performed on a total of 290 cases of B-cell lymphoma, including 92 chronic lymphocytic leukemia/small lymphocytic lymphomas, 53 mantle cell lymphomas, 43 follicular lymphomas, 31 marginal zone 

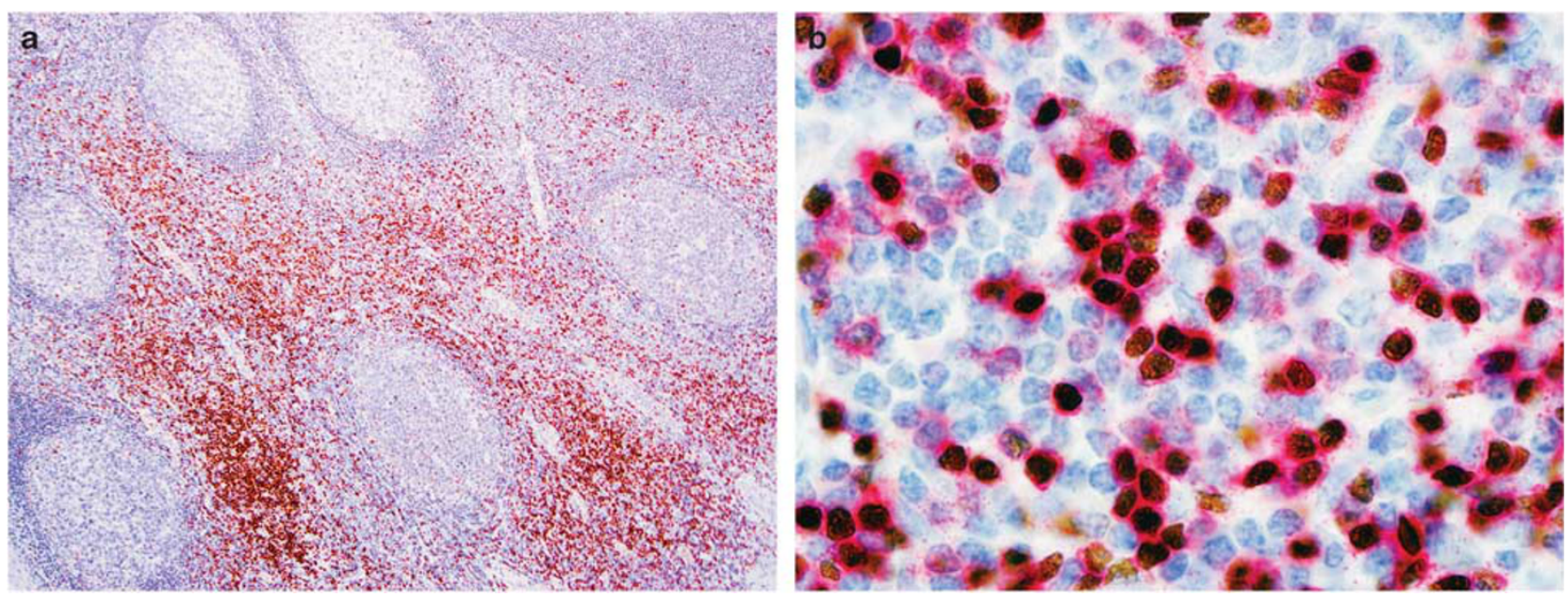

Figure 1 Immunohistochemical staining for LEF1in normal lymphoid tissue. (a) LEF1 positive cells were predominantly in the paracortical regions $(\times 100)$. (b) Double staining of LEF1 (brown) and CD3 (red) demonstrated co-staining of nuclear LEF1 and cytoplasmic CD3 in the same cells $(\times 1000)$.

Table 1 Nuclear overexpression of LEF1 in B-cell lymphomas assessed by immunohistochemistry

\begin{tabular}{|c|c|}
\hline B-cell lymphoma ${ }^{\mathrm{a}}$ & Nuclear overexpression of LEF1 (positive cases/total cases) \\
\hline Chronic lymphocytic leukemia/small lymphocytic lymphoma $(\mathrm{n}=92)$ & 92/92 (100\%), positive in $\sim 100 \%$ cells \\
\hline Without Richter's transformation & 84/84 (100\%; CD5+: 80; CD5-: 2) \\
\hline With Richter's transformation & $8 / 8(100 \%$; all CD5+) \\
\hline Mantle cell lymphoma $(\mathrm{n}=53)$ & $0 / 53$ \\
\hline Classical type & $0 / 47$ \\
\hline Small cell variant & $0 / 2$ \\
\hline Pleomorphic/blastoid variant & $0 / 4$ \\
\hline Marginal zone lymphoma $(\mathrm{n}=31)$ & $0 / 31$ \\
\hline Nodal & 0/15 (CD5-: 13; CD5+: 2) \\
\hline Splenic & 0/3 (CD5-: 2; CD5+: 1) \\
\hline Mucosa-associated lymphoid tissue & 0/13 (all CD5-) \\
\hline \multicolumn{2}{|l|}{ Follicular lymphoma $(\mathrm{n}=43)$} \\
\hline Grade 1-2 & $0 / 31$ \\
\hline Grade 3 & $6 / 12(50 \%)$, positive in $5-15 \%$ cells \\
\hline Diffuse large B-cell lymphoma $(\mathrm{n}=71)$ & 27/71 (38\%), significant staining variability \\
\hline De novo & $23 / 51(45 \%)$ \\
\hline Diffuse large B-cell lymphoma, NOS & $22 / 48$ \\
\hline Primary mediastinal large B-cell lymphoma & $1 / 3$ \\
\hline Transformed from follicular lymphoma & $4 / 17(24 \%)$ \\
\hline Transformed from marginal zone lymphoma & $0 / 2$ \\
\hline Post-transplant lymphoproliferative disorder & $0 / 1$ \\
\hline
\end{tabular}

Abbreviation: LEF1, lymphoid-enhancer-binding factor 1.

${ }^{a}$ Among the cases, three were composite lymphomas, including two cases of composite small lymphocytic lymphoma and follicular lymphoma and one case of composite small lymphocytic lymphoma and mantle cell lymphoma (see results in the text).

lymphomas and 71 diffuse large B-cell lymphomas (Table 1). Of 92 cases of chronic lymphocytic leukemia/small lymphocytic lymphoma (59 formalin-fixed lymph node or other tissue biopsies, 33 B5fixed bone marrow core biopsies), immunostaining for LEF1 demonstrated uniformly strong nuclear staining of LEF1 in virtually all neoplastic cells in 92 of $92(100 \%)$ cases, with relatively greater staining intensity in the prolymphocytes within the proliferation centers (Figure 2a and b). Eight cases contained areas of large cell transformation (Richter's transformation), and the large cells demonstrated the greatest intensity for LEF1 staining in comparison with the background non-transformed cells (Figure 3). Of note, two cases of small lymphocytic lymphomas showed atypical immunophenotype in that the leukemic cells were negative for CD5 by both immunohistochemical staining and 

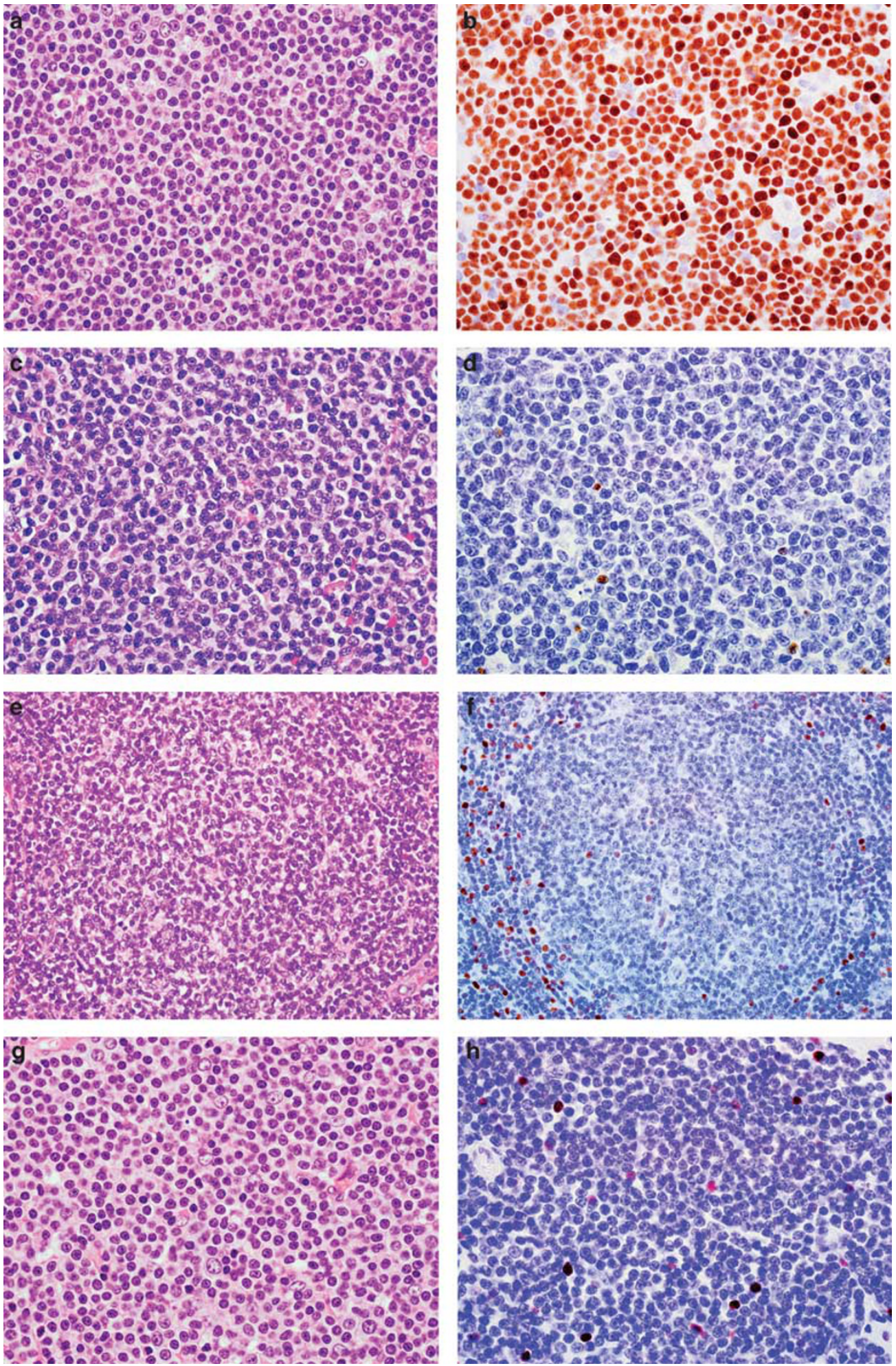

Figure 2 LEF1 expression in small B-cell lymphomas. Left: H\&E staining, right: immunohistochemical staining for LEF1. (a, b) Chronic lymphocytic leukemia/small lymphocytic lymphoma. Uniformly strong nuclear staining of LEF1 was observed in nearly $100 \%$ of neoplastic cells $(\times 600)$. $(\mathbf{c}, \mathbf{d})$ Mantle cell lymphoma. The neoplastic cells were negative for LEF1; rare admixed T cells were positive $(\times 600)$. (e, f) Low grade (grade 1-2) follicular lymphoma. The neoplastic follicles were negative for LEF1; the interfollicular T cells were positive $(\times 400)$. $(\mathbf{g}, \mathbf{h})$ Marginal zone lymphoma. The neoplastic cells were negative for LEF1; the admixed T cells were positive $(\times 600)$.

flow cytometric analysis with three independent anti-CD5 antibodies. However, these two cases did demonstrate morphological features of small lym- phocytic lymphoma, including the presence of proliferation centers. LEF1 staining was positive in nearly all neoplastic cells in these two cases. 

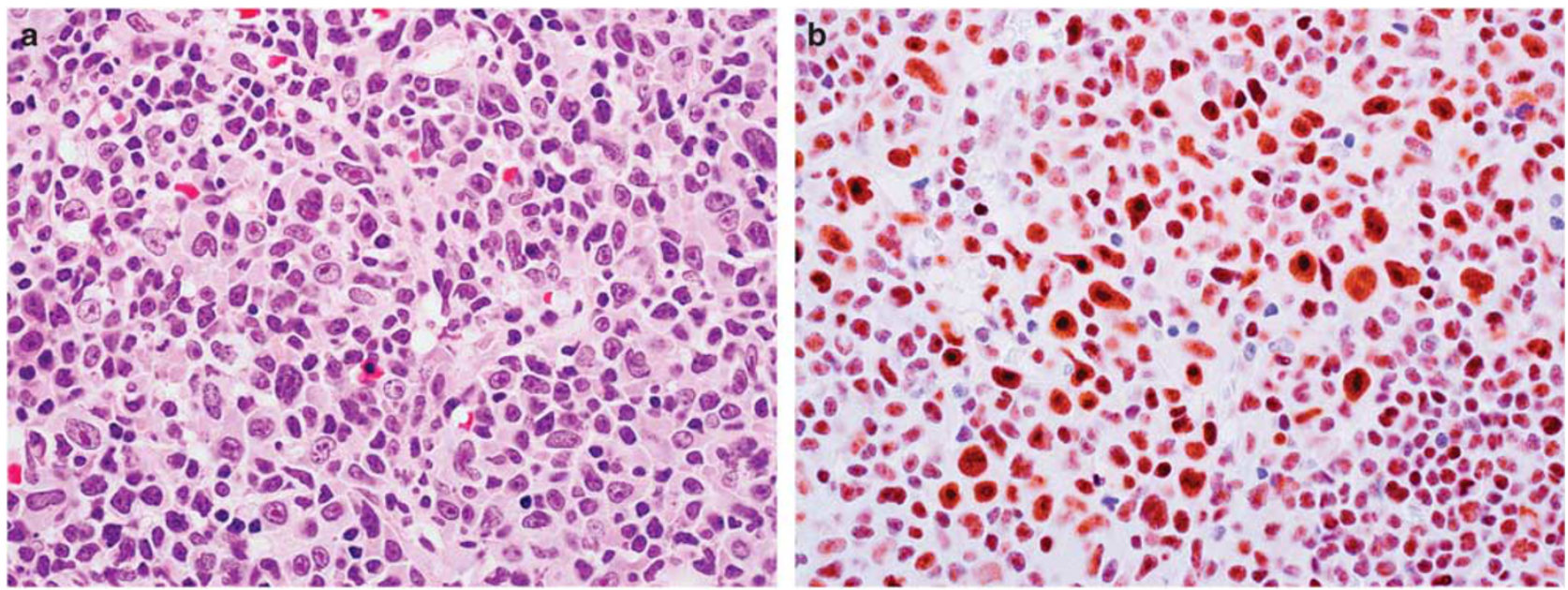

Figure 3 LEF1 expression in Richter's transformation. (a) Chronic lymphocytic leukemia/small lymphocytic lymphoma with Richter's transformation (H\&E stain; $\times 600)$. (b) Immunohistochemistry showed strong nuclear staining of LEF1 in the large transformed cells $(\times 600)$.

The 53 mantle cell lymphomas were composed of 47 cases of common type, 2 small cell variant and 4 aggressive variant (2 pleomorphic and 2 blastoid). The diagnosis of these cases were previously confirmed by immunostaining for cyclin D1 and/or fluorescence in situ hybridization (FISH) or cytogenetic analysis for t(11;14)-CCND1/IgH translocation. ${ }^{18}$ Immunostaining showed that all 53 mantle cell lymphomas were negative for LEF1; positive staining was seen only in the admixed $\mathrm{T}$ cells (Figure 2c and d). Of two cases of small cell variant, one initially presented with marked lymphocytosis in the peripheral blood composed of a monotonous population of small lymphocytes with round nuclei and clumped chromatin, morphologically indistinguishable from chronic lymphocytic leukemia (Figure 4a). Flow cytometric analysis demonstrated a monotypic B-cell population with an immunophenotypic profile (CD20+, CD5 +, CD23+, dim CD79a +, dim FMC-7 + ) compatible with chronic lymphocytic leukemia/small lymphocytic lymphoma although not characteristic. Bone marrow biopsy of the case showed diffuse infiltration of small lymphocytes with morphological features resembling chronic lymphocytic leukemia/small lymphocytic lymphoma (Figure 4b). Immunostaining for LEF1 was completely negative (Figure 4c). The diagnosis of mantle cell lymphoma in this case was established by the positive staining for cyclin D1 (Figure 4d), and confirmed by FISH analysis for $t(11 ; 14)$.

In all 43 follicular lymphomas were included in this study, 31 grade 1-2 (low grade) and 12 grade 3 . In this study, positive staining in $<5 \%$ of neoplastic cells was defined as negative. Immunostaining for LEF1 showed that all 31 cases of low-grade follicular lymphomas were negative; positive staining was seen only in the admixed T cells in the interfollicular areas (Figure 2e and f). Rare weakly positive
B cells were seen within the neoplastic follicles in these low-grade follicular lymphomas, similar to that seen in the normal lymphoid follicles. Of the 12 grade 3 follicular lymphomas, 6 were negative and 6 showed weak to moderate LEF1 staining in a small subset (5-15\%) of neoplastic cells.

A total of 31 marginal zone lymphomas were examined for LEF1 expression in this study, including 15 nodal, 3 splenic and 13 extranodal marginal zone lymphoma of mucosa-associated lymphoid tissue. All 31 cases were negative for LEF1 in the neoplastic cells; positive staining was seen in the admixed $\mathrm{T}$ cells (Figure $2 \mathrm{~g}$ and $\mathrm{h}$ ). Of note, three cases (two nodal and one splenic) were CD5+ marginal zone lymphomas, one of which was initially diagnosed as chronic lymphocytic leukemia/small lymphocytic lymphoma at the referring institution. Review of the lymph node biopsy, including additional immunohistochemistry work up, was consistent with a marginal zone lymphoma. LEF1 was performed and showed that the neoplastic cells were completely negative.

In 71 diffuse large B-cell lymphomas, positive nuclear staining of LEF1 was observed in 27 (38\%) cases. However, the staining intensity and percent of positive cells varied significantly (Figure 5). Nine cases showed positive staining in $10-30 \%$ of neoplastic cells, six in $30-50 \%$ cells, three in $50-70 \%$ cells and nine in $>80 \%$ cells. Of note, LEF1 was positive in 23 of 51 (45\%) de novo diffuse large B-cell lymphomas, including 1 of 3 primary mediastinal large B-cell lymphomas, and 4 of 17 $(24 \%)$ cases transformed from follicular lymphoma. The remaining three cases, two transformed from marginal zone lymphoma and one diffuse large B-cell lymphoma of post-transplant lymphoproliferative disorder, were negative.

Three composite lymphomas were identified in our series, two with composite small lymphocytic 

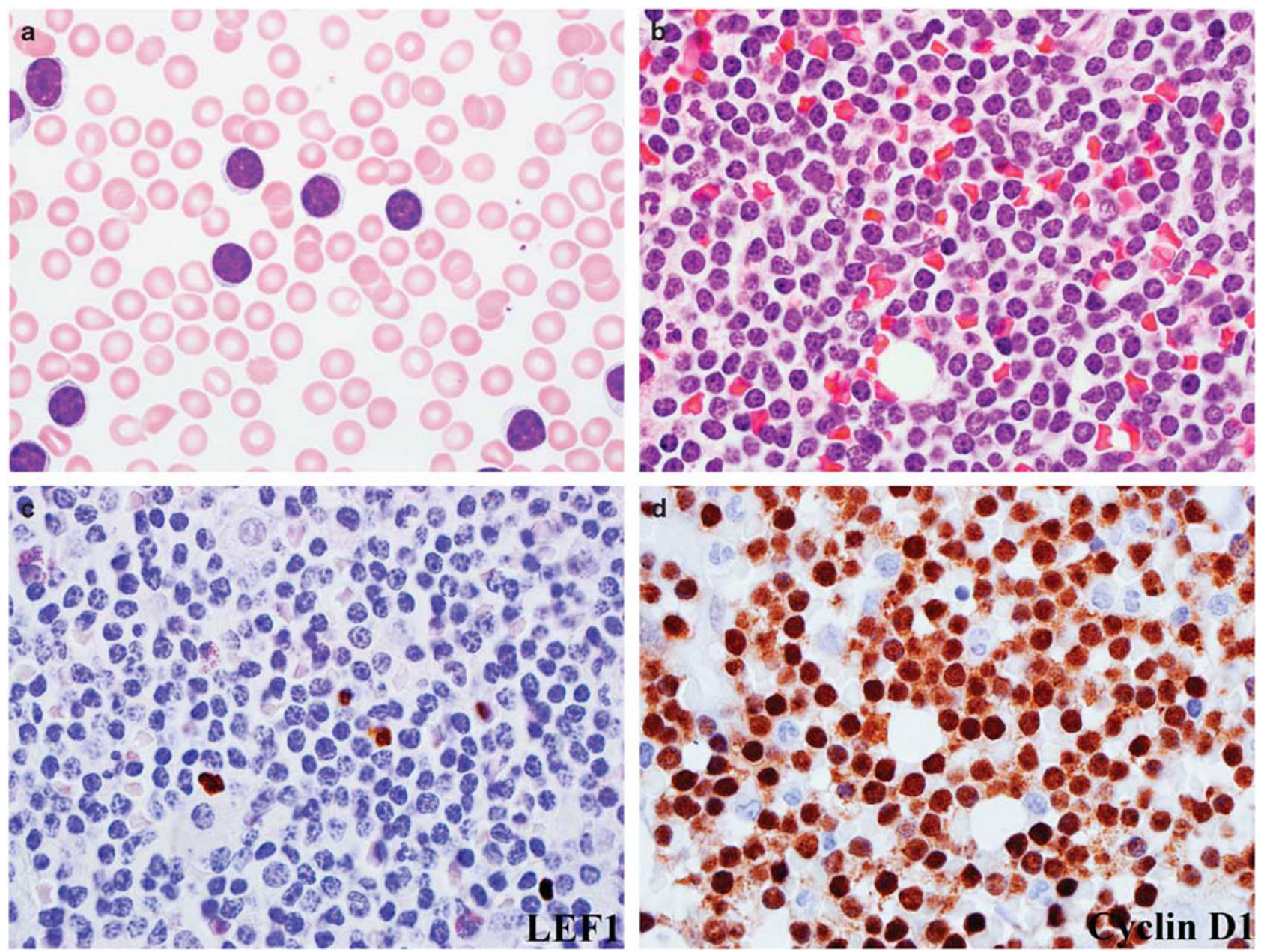

Figure 4 A case of small cell variant of mantle cell lymphoma. (a) The peripheral blood showed marked lymphocytosis composed of a monotonous population of small lymphocytes with round nuclei and clumped chromatin, morphologically indistinguishable from chronic lymphocytic leukemia (Wright-Giemsa stain, $\times 1000$ ). (b) Bone marrow biopsy showed diffuse infiltration of small lymphocytes with round nuclei and clumped chromatin $(H \& E$ stain; $\times 600)$. (c) Immunostaining for LEF1 was negative $(\times 600)$. (d) Immunostaining for cyclin D1 was positive $(\times 600)$.

lymphoma and follicular lymphoma and one with composite small lymphocytic lymphoma and pleomorphic variant of mantle cell lymphoma. In the first two cases, the small lymphocytic lymphoma component was inconspicuous morphologically and was identified by flow cytometric analysis. However, LEF1 staining highlighted the small lymphocytic lymphoma component as patchy LEF1+ $B$ cells between the neoplastic follicles of the follicular lymphoma. In the case of composite small lymphocytic lymphoma and pleomorphic type of mantle cell lymphoma, the latter component consisted of sheets of large pleomorphic lymphoid cells, resembling diffuse large B-cell lymphoma. A diagnosis of mantle cell lymphoma was suggested by a CD5 + monotypic B-cell population detected by flow cytometry, and confirmed by positive cyclin D1 staining in the large cells and fusion of CCND1 and IgH by FISH analysis. However, the geographically distinct small lymphocytic lymphoma component was not recognized until LEF1 was performed on this case. The LEF1 and cyclin D1 staining demonstrated mirror images of the two components in the lymph node biopsy (Figure 6). Further investigation revealed that the patient had a remote history of small lymphocytic lymphoma diagnosed at an outside hospital.

\section{$\beta$-Catenin Expression in Chronic Lymphocytic Leukemia/Small Lymphocytic Lymphoma}

To investigate whether nuclear overexpression of LEF1 in chronic lymphocytic leukemia/small lymphocytic lymphoma represented the involvement of LEF1 in WNT/ $\beta$-catenin signaling in this disease, $\beta$-catenin expression was assessed by immunohistochemistry in 50 cases of chronic lymphocytic leukemia/small lymphocytic lymphoma (25 bone marrow and 25 lymph node biopsies).

In normal lymphoid tissue, positive $\beta$-catenin staining was seen as perinuclear dots in the 

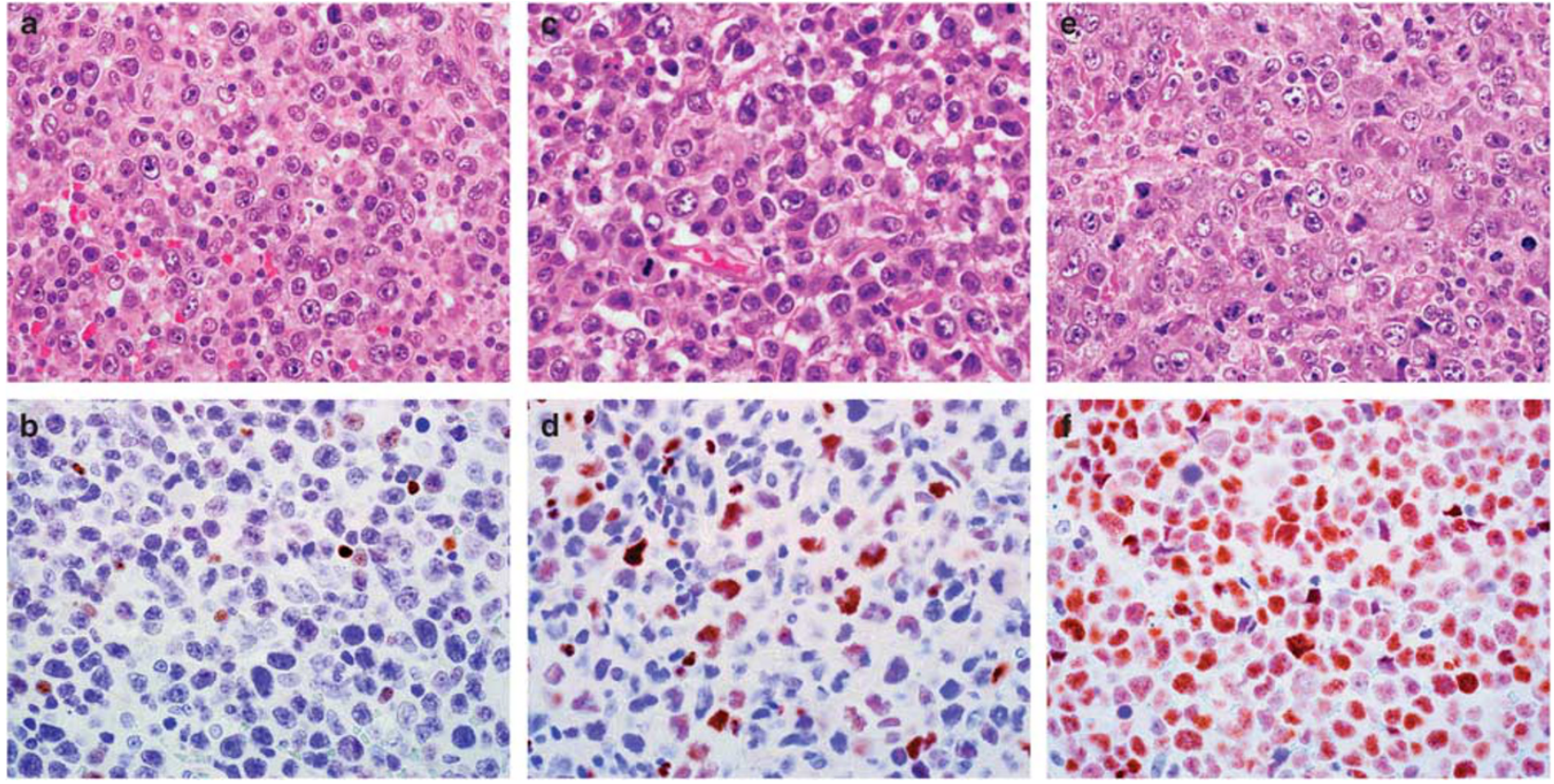

Figure 5 LEF1 expression in diffuse large B-cell lymphoma. Upper: H\&E stain ( $\times 600)$; lower: immunohistochemical staining for LEF1 $(\times 600)$. Diffuse large B-cell lymphomas showed significant variability in LEF1 staining from completely negative (a, b), positive in a subset of cells $(\mathbf{c}, \mathbf{d})$, to positive in the vast majority of cells $(\mathbf{e}, \mathbf{f})$.

lymphocytes, and membranous staining in the squamous mucosa in tonsillar tissue (Figure 7). Of 50 cases of chronic lymphocytic leukemia/small lymphocytic lymphoma, $44(88 \%)$ were negative for nuclear staining of $\beta$-catenin and the positive staining was restricted to the cytoplasm (Figure 8). The remaining six $(12 \%)$ cases showed moderate nuclear staining for $\beta$-catenin in $10-20 \%$ cells in four cases, $30 \%$ in one case and $60 \%$ in one case. Of four cases of chronic lymphocytic leukemia/small lymphocytic lymphoma with Richter's transformation, one was positive for $\beta$-catenin in about $15 \%$ of cells.

\section{Discussion}

Gene expression profiling has demonstrated multiple genes that are differentially expressed in chronic lymphocytic leukemia vs normal B cell; LEF1 is one of the genes identified and is expressed over 800fold higher in chronic lymphocytic leukemia than in normal B cells. ${ }^{16,17}$ A recent study shows that LEF1 is not only overexpressed in chronic lymphocytic leukemia, but also in the CD5 + /CD19 + cells from patients with monoclonal B-cell lymphocytosis, suggesting a role of LEF1 in early leukemogenesis of chronic lymphocytic leukemia. ${ }^{7}$ So far, the data on LEF1 expression in B-cell lymphoma are limited, and the evaluation has been through molecular or other methods primarily used in research settings. Our study assessed LEF1 expression by immunohistochemistry in a large series of chronic lymphocytic leukemia/small lymphocytic lymphoma as well as other types of B-cell lymphomas. We also examined $\beta$-catenin expression in chronic lymphocytic leukemia/small lymphocytic lymphoma to investigate whether overexpression of LEF1 has a role in pathogenesis of the disease by mediating $\mathrm{WNT} / \beta$-catenin signaling.

Our study showed uniformly strong nuclear staining of LEF1 in virtually all neoplastic cells in 92 of 92 chronic lymphocytic leukemia/small lymphocytic lymphomas, but not in other types of small B-cell lymphomas examined, including 53 common type and variant mantle cell lymphomas, 33 low-grade (grade 1-2) follicular lymphomas and 31 marginal zone lymphomas of various tissue origins; positive staining was seen only in the admixed T cells. In grade 3 follicular lymphomas, LEF1 staining was seen only in a small subset of neoplastic cells (5-15\%) in 6 of 12 cases. Our results indicate that nuclear overexpression of LEF1 is readily detectable by immunohistochemistry in paraffin-embedded tissue and is highly associated with chronic lymphocytic leukemia/small lymphocytic lymphoma among small B-cell lymphomas. Thus, LEF1 may serve as a convenient immunohistochemical marker for diagnosis and differential diagnosis of chronic lymphocytic leukemia/small lymphocytic lymphoma, particularly when morphological features or immunophenotype is atypical or unavailable.

The diagnosis of chronic lymphocytic leukemia/ small lymphocytic lymphoma has been primarily based on the morphological evaluation and immunophenotyping; there has been no specific immunohistochemical marker available. However, 

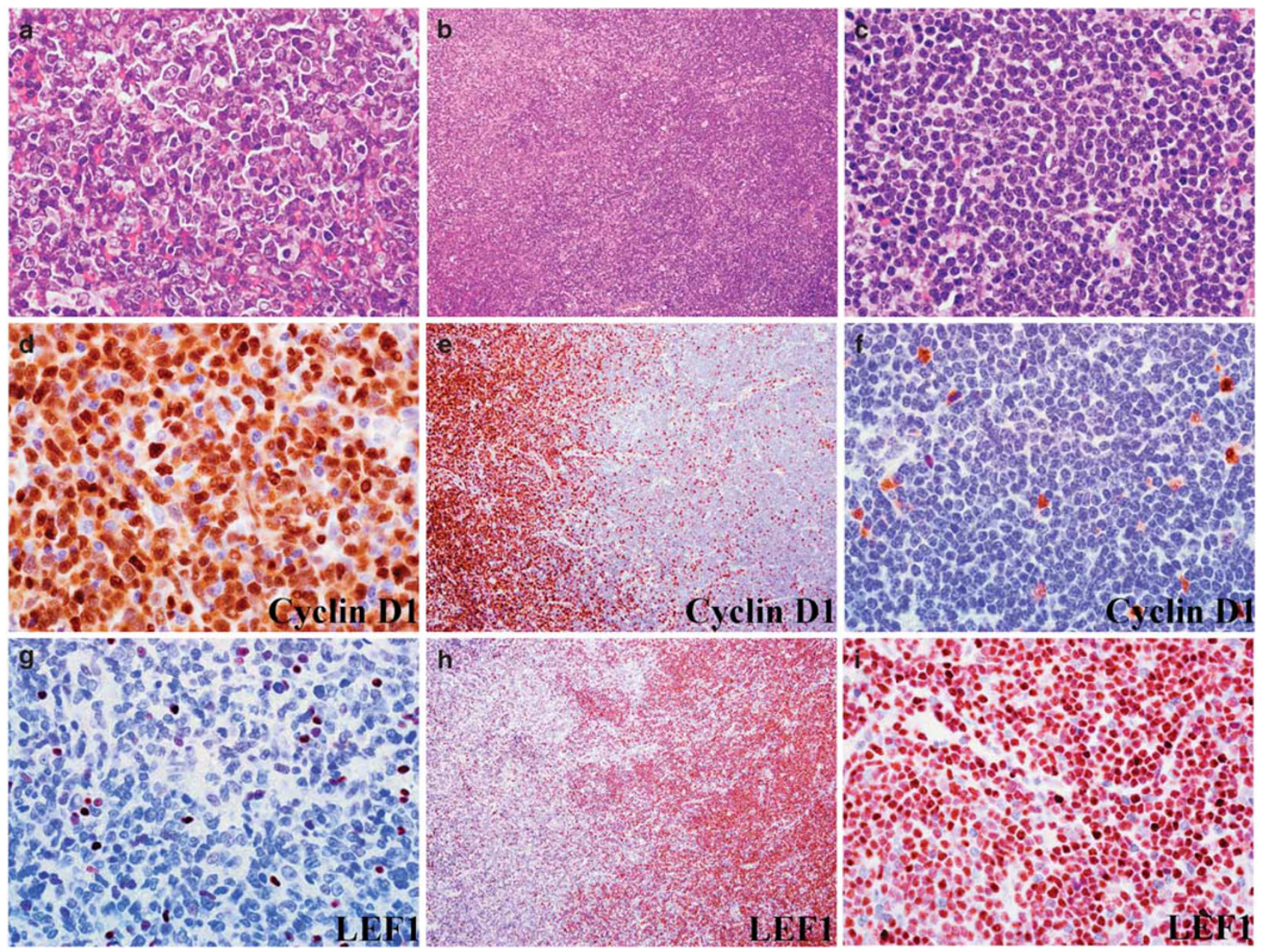

Figure 6 Composite lymphoma composed of small lymphocytic lymphoma and mantle cell lymphoma, pleomorphic type. Middle (b, e, h): low-power view of the lymph node biopsy $(\times 100)$; left $(\mathbf{a}, \mathbf{d}, \mathbf{g})$ : high-power view of the mantle cell lymphoma component $(\times 600)$; right $(\mathbf{c}, \mathbf{f}, \mathbf{i})$ : high-power view of the small lymphocytic lymphoma component $(\times 600)$. (a-c) Two distinct areas composed of large pleomorphic cells on the left and small lymphocytes on the right (H\&E stain). (d-f) Immunostaining for cyclin D1 showed nuclear positivity in the large cell component on the left, consistent with mantle cell lymphoma. The small cell component on the right was negative. (g-i) Immunostaining for LEF1 demonstrated nuclear positivity in the small cell component (small lymphocytic lymphoma) on the right, but negative in the large cell component (mantle cell lymphoma) on the left.

significant overlap in immunophenotype between chronic lymphocytic leukemia/small lymphocytic lymphoma and mantle cell lymphoma, the two major types of CD5 + B-cell lymphomas, is well recognized. ${ }^{19,20}$ CD5 expression in other B-cell lymphomas, such as marginal zone lymphoma and follicular lymphoma, has also been reported. ${ }^{21-26}$ In addition, rare cases of chronic lymphocytic leukemia/small lymphocytic lymphoma may be CD5-. ${ }^{1}$ These atypical immunophenotypes may cause diagnostic confusion, particularly when the morphological features are not classical.

The utility of LEF1 in diagnosis and differential diagnosis of chronic lymphocytic leukemia/small lymphocytic lymphoma has been documented in several interesting cases in this study, including two cases of CD5 - chronic lymphocytic leukemia/small lymphocytic lymphoma, a small cell variant of mantle cell lymphoma identified initially in the peripheral blood with morphological features indistinguishable from chronic lymphocytic leukemia, one of the three cases of CD5 + marginal zone lymphoma initially misdiagnosed as small lymphocytic lymphoma and two of the three cases of composite lymphoma (small lymphocytic lymphoma and follicular lymphoma) in which the small lymphocytic lymphoma detected by flow cytometry was unable to be confirmed by morphological evaluation. In the remaining case of composite lymphoma (small lymphocytic lymphoma and pleomorphic variant of mantle cell lymphoma), the small lymphocytic lymphoma was not recognized until immunostaining for LEF1 was performed in this study. We expect that LEF1, in combination with SOX11, may be particularly important in the differential diagnosis of cyclin D1-negative mantle cell lymphoma vs chronic lymphocytic leukemia/ small lymphocytic lymphoma. ${ }^{27}$ Two cases of 

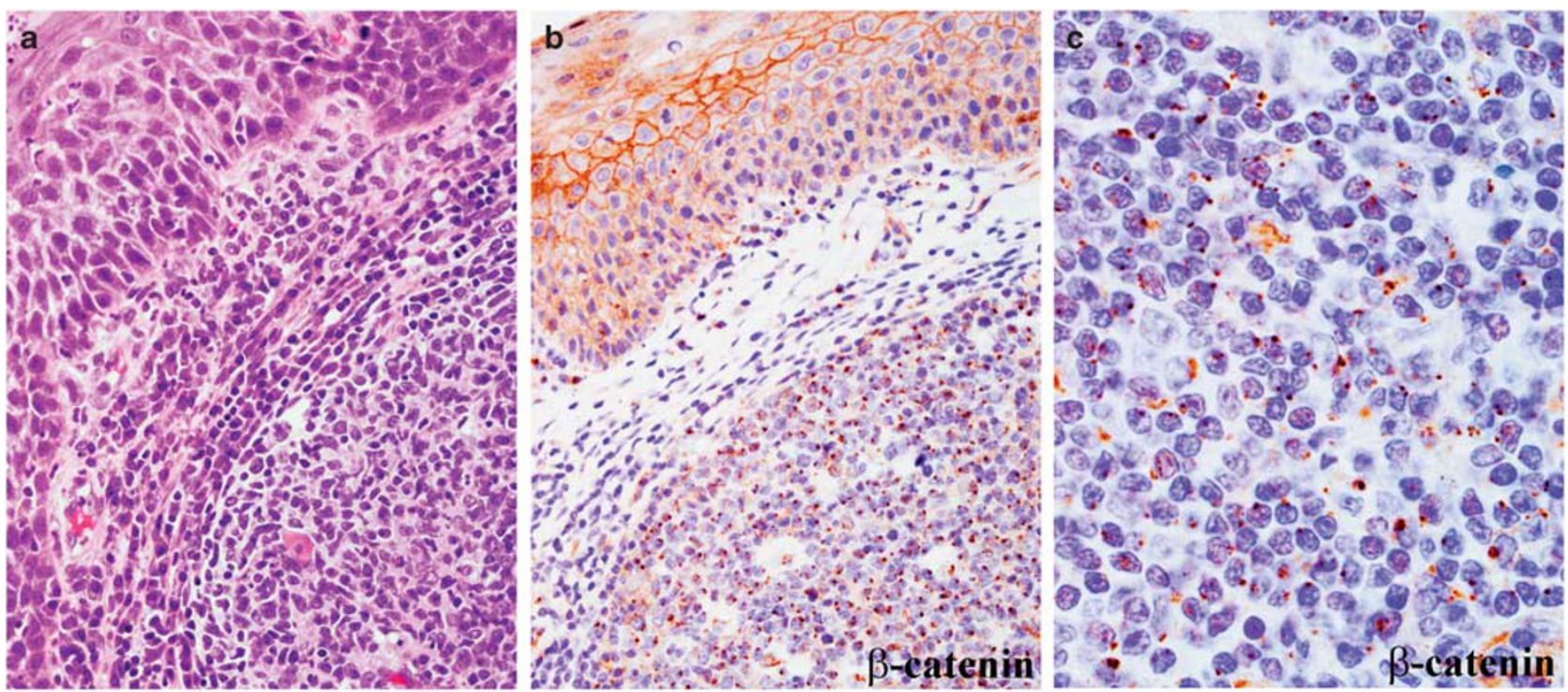

Figure 7 Immunohistochemical staining for $\beta$-catenin in normal lymphoid tissue. (a) Tonsil (H\&E stain; $\times 400)$. (b) Immunohistochemistry for $\beta$-catenin showed perinuclear dot-like staining in the lymphocytes and membranous staining in the squamous mucosa in the tonsillar tissue $(\times 400)$. (c) High-power view of the the perinuclear dot-like staining of $\beta$-catenin in normal lymphocytes; the nuclei were negative $(\times 1000)$.
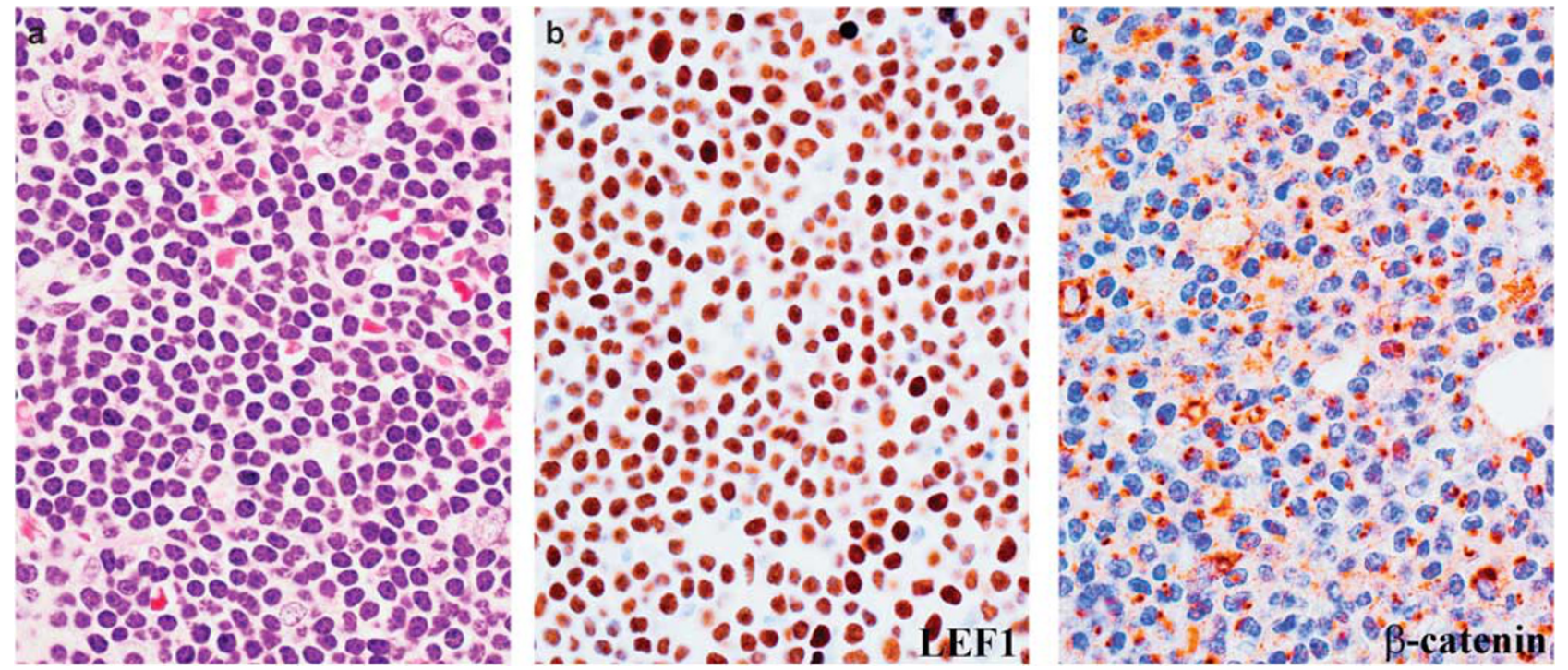

Figure $8 \beta$-Catenin expression in chronic lymphocytic leukemia/small lymphocytic lymphoma. (a) Leukemic infiltrate in a bone marrow biopsy (H\&E stain; $\times 1000)$. (b) Immunostaining for LEF1 showed strong nuclear staining in virtually all neoplastic lymphocytes $(\times 1000)$. (c) Immunostaining for $\beta$-catenin showed no nuclear staining. The positive staining was seen only in the cytoplasm as perinuclear dots, similar to normal lymphocytes $(\times 1000)$.

chronic lymphocytic leukemia/small lymphocytic lymphomas in our series were CD5- by both flow cytometric analysis and immunohistochemistry; however, they demonstrated typical morphological features of small lymphocytic lymphoma including the presence of multiple proliferation centers. LEF1 was diffusely positive in both cases, similar to the CD5 + chronic lymphocytic leukemia/small lymphocytic lymphomas. CD5 is a key marker for the diagnosis of chronic lymphocytic leukemia/small lymphocytic lymphoma, and lack of CD5 staining often raises question about the diagnosis. However, rare cases of CD5- chronic lymphocytic leukemia/ small lymphocytic lymphoma do exist. ${ }^{1}$ Our results indicate that LEF1 may be a valuable marker to aid in the diagnosis of CD5- chronic lymphocytic leukemia/small lymphocytic lymphoma.

Unlike small B-cell lymphomas, however, diffuse large B-cell lymphoma demonstrated significant variability in LEF1 staining, from completely negative to positive in $>80 \%$ of cells, which added additional evidence to the known biological hetero- 
geneity of diffuse large B-cell lymphoma. Interestingly, the de novo large B-cell lymphomas demonstrated higher incidence of positivity for LEF1 (23 of $51 ; 45 \%$ ) than cases transformed from follicular lymphoma (4 of 17; 24\%). The biological and clinical significance of LEF1 in diffuse large B-cell lymphoma is currently under investigation.

The function of LEF1 in chronic lymphocytic leukemia is not well understood. It has been shown that knockdown of LEF1 by siRNA decreases the survival of chronic lymphocytic leukemia cells in vitro. There are also several lines of evidence indicating that WNT signaling is constitutively activated in chronic lymphocytic leukemia., ${ }^{3,7}$ Given the central role of LEF1 in the canonical WNT signaling, it is reasonable to hypothesize that LEF1 may have a pro-survival role in chronic lymphocytic leukemia through WNT/ $\beta$-catenin signaling. The ultimate mediator of this pathway is the nuclear complex composed of LEF/TCF and $\beta$-catenin that function together to stimulate the transcription of target genes involved in cell proliferation and survival. In the absence of a WNT ligand, $\beta$-catenin is phosphorylated by a 'destruction complex' and degraded in the cytoplasm. When a WNT ligand binds to its receptor, the destruction complex is disassembled and results in an accumulation and nuclear translocation of $\beta$-catenin, where it binds to $\mathrm{LEF} / \mathrm{TCF}$ to regulate the transcription of WNT target genes. Immunohistochemistry is a very useful tool to assess subcellular localization of $\beta$-catenin.

Although all chronic lymphocytic leukemia/small lymphocytic lymphoma examined in this study demonstrated strong nuclear overexpression of LEF1 in virtually all neoplastic cells, 44 of 50 cases $(88 \%)$ examined for $\beta$-catenin expression were negative for nuclear staining. The remaining six $(12 \%)$ cases exhibited moderate nuclear staining of $\beta$-catenin in variable numbers of cells. A previous study evaluated $\beta$-catenin levels by western blot and found no increase in the total $\beta$-catenin protein in chronic lymphocytic leukemia in comparison to normal B cell. ${ }^{7}$ The lack of nuclear localization of $\beta$-catenin in the majority of chronic lymphocytic leukemia/small lymphocytic lymphomas as demonstrated in our study and no increase in the total $\beta$-catenin protein in the leukemic cells vs normal $\mathrm{B}$ cells as shown by others argue against a primary role of LEF1 in chronic lymphocytic leukemia as a mediator of $\mathrm{WNT} / \beta$-catenin signaling. Although LEF1 is best known for its role in $\mathrm{WNT} / \beta$-catenin signaling, there is evidence that it can also function independent of $\beta$-catenin in transcription regulation, and these functions are induced by the HMG domain or by its interaction with alternative cofactors. ${ }^{28-31}$ It will be of great interest to assess these alternative mechanisms of action of LEF1 in chronic lymphocytic leukemia. Recently, several pharmacological inhibitors or small molecules have been shown to efficiently induce apoptosis of chronic lymphocytic leukemia cells in vitro through their interaction with LEF1. ${ }^{32,33}$ These findings implicate that LEF1 may be a potential therapeutic target in the future for patients with chronic lymphocytic leukemia.

In summary, this study demonstrates that nuclear overexpression of LEF1 assessed by immunohistochemistry is highly associated with chronic lymphocytic leukemia/small lymphocytic lymphoma among small B-cell lymphomas and may serve as a useful marker for diagnosis and differential diagnosis of the disease. Universal expression of LEF1 in chronic lymphocytic leukemia/small lymphocytic lymphoma but lack of nuclear localization of $\beta$-catenin in the majority of the cases suggests that the pro-survival effect of LEF1 in chronic lymphocytic leukemia may be independent of $\mathrm{WNT} / \beta$ catenin signaling.

\section{Acknowledgement}

We thank Chunyan Luan (Pathology Core Facilities, Robert H Lurie Comprehensive Cancer Center of Northwestern University) for her excellent work with the immunohistochemistry.

\section{Disclosure/conflict of interest}

The authors declare no conflict of interest.

\section{References}

1 Muller-Hermelink HK, Montserrat E, Catovsky D, et al. Chronic lymphocytic leukemia/small lymphocytic lymphoma. In: Swerdlow SH, Campo E, Harris NL, Jaffe ES, Pileri SA, Stein H, Thiele J, Vardiman JW (eds). WHO Classification of Tumours of Haematopoietic and Lymphoid Tissues. IARC: Lyon, France, 2008, pp 180-182.

2 Damle RN, Calissano C, Chiorazzi N. Chronic lymphocytic leukaemia: a disease of activated monoclonal B cells. Best Pract Res Clin Haematol 2010;23:33-45.

$3 \mathrm{Lu} \mathrm{D}$, Zhao Y, Tawatao R, et al. Activation of the Wnt signaling pathway in chronic lymphocytic leukemia. Proc Natl Acad Sci USA 2004;101:3118-3123.

4 Howe D, Bromidge T. Variation of LEF-1 mRNA expression in low-grade B-cell non-Hodgkin's lymphoma. Leuk Res 2006;30:29-32.

5 Memarian A, Hojjat-Farsangi M, Asgarian-Omran H, et al. Variation in WNT genes expression in different subtypes of chronic lymphocytic leukemia. Leuk Lymphoma 2009;50:2061-2070.

$6 \mathrm{Wu}$ QL, Zierold C, Ranheim EA. Dysregulation of Frizzled 6 is a critical component of B-cell leukemogenesis in a mouse model of chronic lymphocytic leukemia. Blood 2009;113:3031-3039.

7 Gutierrez Jr A, Tschumper RC, Wu X, et al. LEF-1 is a prosurvival factor in chronic lymphocytic leukemia and is expressed in the preleukemic state of monoclonal B-cell lymphocytosis. Blood 2010;116: 2975-2983. 
8 Novak A, Dedhar S. Signaling through beta-catenin and Lef/Tcf. Cell Mol Life Sci 1999;56:523-537.

9 Eastman Q, Grosschedl R. Regulation of LEF-1/TCF transcription factors by Wnt and other signals. Curr Opin Cell Biol 1999;11:233-240.

10 Moon RT, Kohn AD, De Ferrari GV, et al. Wnt and $\beta$ catenin signaling: disease and therapies. Nat Rev Genet 2004;5:691-701.

11 Reya T, O’Riordan M, Okamura R, et al. Wnt signaling regulates B lymphocyte proliferation through a LEF-1 dependent mechanism. Immunity 2000;13:15-24.

12 Reya T, Okamura R, Grosschedl R. Control of lymphocyte differentiation by the LEF-1/TCF family of transcription factors. Cold Spring Harb Symp Quant Biol 1999;64:133-140.

13 Staal FJ, Sen JM. The canonical Wnt signaling pathway plays an important role in lymphopoiesis and hematopoiesis. Eur J Immunol 2008;38:1788-1794.

14 Petropoulos K, Arseni N, Schessl C, et al. A novel role for Lef-1, a central transcription mediator of Wnt signaling, in leukemogenesis. J Exp Med 2008;205: $515-522$.

15 Dosen G, Tenstad E, Nygren MK, et al. Wnt expression and canonical Wnt signaling in human bone marrow B lymphopoiesis. BMC Immunol 2006;7:13.

16 Klein U, Tu Y, Stolovitzky GA, et al. Gene expression profiling of $\mathrm{B}$ cell chronic lymphocytic leukemia reveals a homogeneous phenotype related to memory B cells. J Exp Med 2001;194:1625-1638.

17 Jelinek DF, Tschumper RC, Stolovitzky GA, et al. Identification of a global gene expression signature of B-chronic lymphocytic leukemia. Mol Cancer Res 2003;1:346-361.

18 Chen YH, Gao J, Fan G, et al. Nuclear expression of sox11 is highly associated with mantle cell lymphoma but is independent of $\mathrm{t}(11 ; 14)(\mathrm{q} 13 ; \mathrm{q} 32)$ in non-mantle cell B-cell neoplasms. Mod Pathol 2010;23:105-112.

19 Gao J, Peterson L, Nelson B, et al. Immunophenotypic variations in mantle cell lymphoma. Am J Clin Pathol 2009;132:699-706.

20 Ho AK, Hill S, Preobrazhensky SN, et al. Small B-cell neoplasms with typical mantle cell lymphoma immunophenotypes often include chronic lymphocytic leukemias. Am J Clin Pathol 2009;131:27-32.

21 Vassallo J, Bousquet M, Quelen C, et al. CD5-positive diffuse large B cell lymphoma arising from a CD5positive follicular lymphoma. J Clin Pathol 2007;60: $573-575$.
22 Tasaki K, Shichishima A, Furuta M, et al. CD5-positive mucosa-associated lymphoid tissue (MALT) lymphoma of ocular adnexal origin: usefulness of fluorescence in situ hybridization for distinction between mantle cell lymphoma and MALT lymphoma. Pathol Int 2007;57:101-107.

23 Dronca RS, Jevremovic D, Hanson CA, et al. CD5-positive chronic B-cell lymphoproliferative disorders: diagnosis and prognosis of a heterogeneous disease entity. Cytometry B Clin Cytom 2010;78(Suppl 1):S35-S41.

24 Baseggio L, Traverse-Glehen A, Petinataud F, et al. CD5 expression identifies a subset of splenic marginal zone lymphomas with higher lymphocytosis: a clinicopathological, cytogenetic and molecular study of 24 cases. Haematologica 2010;95:604-612.

25 Barry TS, Jaffe ES, Kingma DW, et al. CD5+ follicular lymphoma: a clinicopathologic study of three cases. Am J Clin Pathol 2002;118:589-898.

26 Tiesinga JJ, Wu CD, Inghirami G. CD5+ follicle center lymphoma. Immunophenotyping detects a unique subset of 'floral' follicular lymphoma. Am J Clin Pathol 2000;114:912-921.

27 Mozos A, Royo C, Hartmann E, et al. SOX11 expression is highly specific for mantle cell lymphoma and identifies the cyclin D1-negative subtype. Haematologica 2009;94:1555-1562.

28 Arce L, Yokoyama NN, Waterman ML. Diversity of LEF/TCF action in development and disease. Oncogene 2006;25:7492-7504.

29 Ross DA, Kadesch T. The notch intracellular domain can function as a coactivator for LEF-1. Mol Cell Biol 2001;21:7537-7544.

30 Bruhn L, Munnerlyn A, Grosschedl R. ALY, a contextdependent coactivator of LEF-1 and AML-1, is required for TCRalpha enhancer function. Genes Dev 1997;11:640-653.

31 Chen QY, Zhang T, Pincus SH, et al. Human CD1D gene expression is regulated by LEF-1 through distal promoter regulatory elements. J Immunol 2010;184: 5047-5054.

$32 \mathrm{Lu} \mathrm{D}$, Liu JX, Endo T, et al. Ethacrynic acid exhibits selective toxicity to chronic lymphocytic leukemia cells by inhibition of the Wnt/beta-catenin pathway. PLoS One 2009;4:e8294.

33 Gandhirajan RK, Staib PA, Minke K, et al. Small molecule inhibitors of Wnt/ $\beta$-catenin/Lef-1 signaling induces apoptosis in chronic lymphocytic leukemia cells in vitro and in vivo. Neoplasia 2010;12:326-335. 